\title{
Elevación de piso de seno maxilar con técnica de ventana lateral y colocación simultánea de implantes: reporte de un caso.
}

\section{Sinus floor elevation with a lateral window technique and simultaneous dental implants placement: case report.}

\begin{abstract}
Dulce Bustillo $^{1^{*}}$, Maya Zuloaga ${ }^{2}$
1. Estudiante de posgrado de periodoncia de la Universidad Tecnológica de México, México. 2. Docente del posgrado de periodoncia de la Universidad Tecnológica de México, México.

RESUMEN

La colocación de implantes en la zona posterior maxilar resulta en numerosos desafíos para la rehabilitación fija implanto-soportada debido a la falta de reborde residual . La disponibilidad ósea de la región posterior del maxilar se ve reducida por varios factores, entre ellos, la pérdida prematura de dientes, presencia de enfermedad periodontal, o hiperneumatización de las cavidades sinusales, posterior a exodoncias de la zona posterior. El presente artículo reporta un caso de una paciente femenina de 51 años, con disminución de altura del reborde alveolar en la zona de premolares, describiendo la técnica de elevación de piso de seno maxilar por medio de la realización de una ventana lateral y la colocación simultánea de 2 implantes
\end{abstract}

PALABRAS CLAVE

Reborde alveolar, Seno maxilar, Implantes, Hiperneumatización

Rev. Clin. Periodoncia Implantol. Rehabil. Oral Vol. 10(3); 159-162, 2017.

\section{ABSTRACT}

The edentulous posterior maxillary implant presents numerous challenges that are unique to this region. The bone availability of the posterior maxilla is reduced, among other things, because of the premature loss of teeth, presence of periodontal disease, or hiperpneumatization of the sinus cavities after extractions of posterior teeth. This article presents a case report of a 51 years old female patient with the alveolar ridge diminished at premolars zone, describing the surgical technique of sinus floor elevation with a lateral window and the simultaneous colocation of 2 dental implants.

KEYWORDS

Alveolar ridge, Maxillary sinus, Dental implants, Hiperneumatization

Rev. Clin. Periodoncia Implantol. Rehabil. Oral Vol. 10(3); 159-162, 2017.

\section{INTRODUCCIÓN}

El uso de implantes dentales para la rehabilitación fija de pacientes parcialmente desdentados tiene resultados muy predecibles a largo plazo en casos con una densidad y volumen óseo adecuado para tener una adecuada estabilidad de los implantes. En presencia de un edentulismo del maxilar posterior, la cresta ósea alveolar disminuye debido a la atrofia ósea, dando lugar a la neumatización del seno maxilar.

En este caso la colocación de implantes en zonas atróficas, junto con la reabsorción del reborde maxilar superior, la neumatización de los senos maxilares y la calidad ósea, son factores que pueden dificultar la colocación de implantes dentales y por lo tanto la rehabilitación protésica.

El seno maxilar es una cavidad triangular de forma piramidal cuya base es interna y corresponde a la pared externa de las fosas nasales y su vértice externo se corresponde con el hueso malar.

Es el más grande de los senos paranasales; su tamaño es inversamente proporcional al tamaño de la fosa canina, y está tapizado por la membrana de Schneider la cual contiene un epitelio cilíndrico simple seudoestratificado ciliado con escasas glándulas mucosas. Tiene tres caras: anterior o yugal, posterior o pterigomaxilar y superior, con dimensiones aproximadas de $3 \mathrm{~cm}$ de fondo en sentido antero porsterior, $2.5 \mathrm{~cm}$ de anchura y $3.75 \mathrm{~cm}$ de altura; con un volumen medio de $15 \mathrm{~cm}^{3}$

La elevación de piso de seno maxilar representa el abordaje quirúrgico más comúnmente utilizado para superar los problemas de neumatización, ya que permite la colocación de implantes de altura convencional en áreas de cantidades insuficientes de hueso. Este procedimiento se puede realizar en uno o dos tiempos quirúrgicos dependiendo de la cantidad de reborde residual. Si el reborde residual de igual o mayor a $5 \mathrm{~mm}$ se puede realizar la elevación del piso del seno con la colocación simultánea de los implantes ${ }^{(1)}$.

La elevación del piso del seno maxilar, puede realizarse de acuerdo a dos técnicas. La primera con abordaje quirúrgico a través de una osteotomía lateral del seno maxilar y la segunda mediante abordaje transalveolar.

Basado en la literatura y de acuerdo a estudios realizados como el de Fugazzoto y Vlassis en 1998 con un seguimiento a 6 años en 222 elevaciones de seno con 510 implantes $^{(2)}$ y Jurisic et al ${ }^{(3)}$ con un seguimiento a 3 años en 61 elevaciones de seno y 80 implantes valorando ambas técnicas de abordaje quirúrgico se obtuvieron tasas de éxito similares, justificando la tendencia actual de que no existe diferencia significativa del éxito implantológico en relación a la técnica de abordaje utilizada.

El propósito de la presentación de este caso fue describir la técnica quirúrgica de la elevación del piso de seno maxilar con la técnica de ventana lateral utilizando el kit SLA de NeoBiotech para la colocación posterior de dos implantes dentales.

\section{MATERIAL Y MÉTODO}

Paciente de 51 años de edad, de sexo femenino que acude a la clínica del posgrado de periodoncia de la facultad de ondontología de la UNITEC para la restauración de una zona edéntula posterior de lado izquierdo. 
Paciente sin compromiso sistémico y con un diagnóstico periodontal de gingivitis asociada a placa. (fig 1a ) Al examen clínico y radiográfico se evidencia la ausencia de los dientes 24,25 y 26 con la neumatización de seno maxilar en esa zona. Se realizó un análisis tomográfico mostrando un reborde residual de $4.6 \mathrm{~mm}$ en sentido apico-coronal. (Fig $1 \mathrm{~b}$ y $1 \mathrm{c}$ )
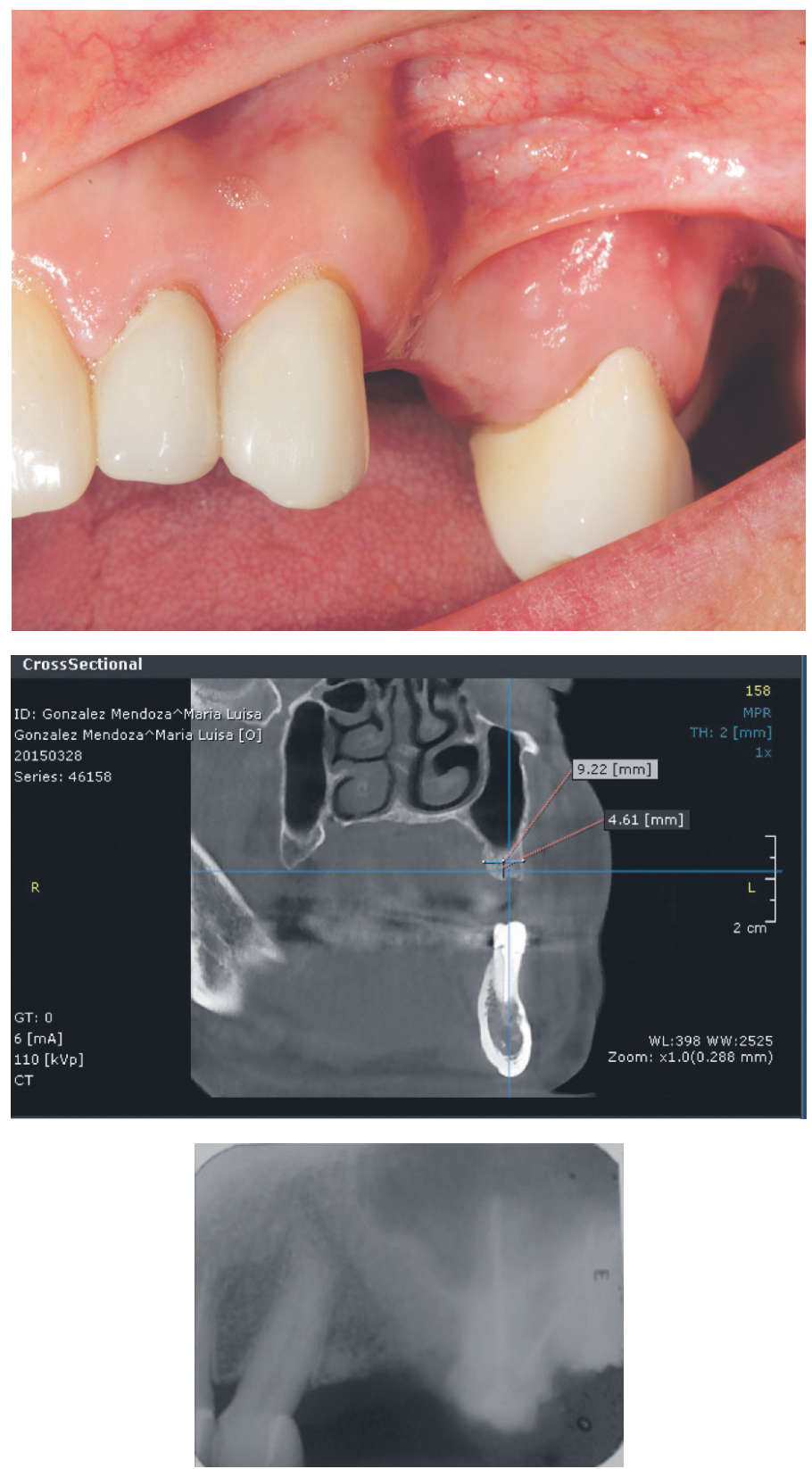

Figura 1. Fig1a Fotografía lateral de la zona que muestra la ausencia de los dientes 24 y 25 . Fig 1b: Radiografía inicial mostrando la neumatización del piso de seno maxilar. Fig 1c: Se muestra el análisis tomográfico con una distancia de reborde residual de $4.6 \mathrm{~mm}$

Se planificó la elevación de seno maxilar con técnica de ventana lateral utilizando el kit SLA NeoBiotech, con la colocación de un materia regenerativo de tipo xenoinjerto para una posterior rehabilitación protésica fija atornillada. En el procedimiento se utilizó anestesia infiltrativa local con dos cartuchos de lidocaína al $2 \%$ con vasoconstrictor. Se realizó una incisión supracrestal y el acceso a la pared antrolateral del seno maxilar se logró mediante la elevación de un colgajo trapezoidal de espesor total. (Figura 2a )

Después de elevar el colgajo, se procedió a fresar para la colocación del implante que sustituirá el diente 24 para posteriormente realizar la osteotomía para delimitar la ventana ósea con el kit previamente descrito, el motor quirúrgico a 1,000 RPM como especifica la casa comercial y constante irrigación.( Figura 2b)

Se utilizó la fresa LS-reamer de $5.5 \mathrm{~mm}$ de diámetro para realizar la
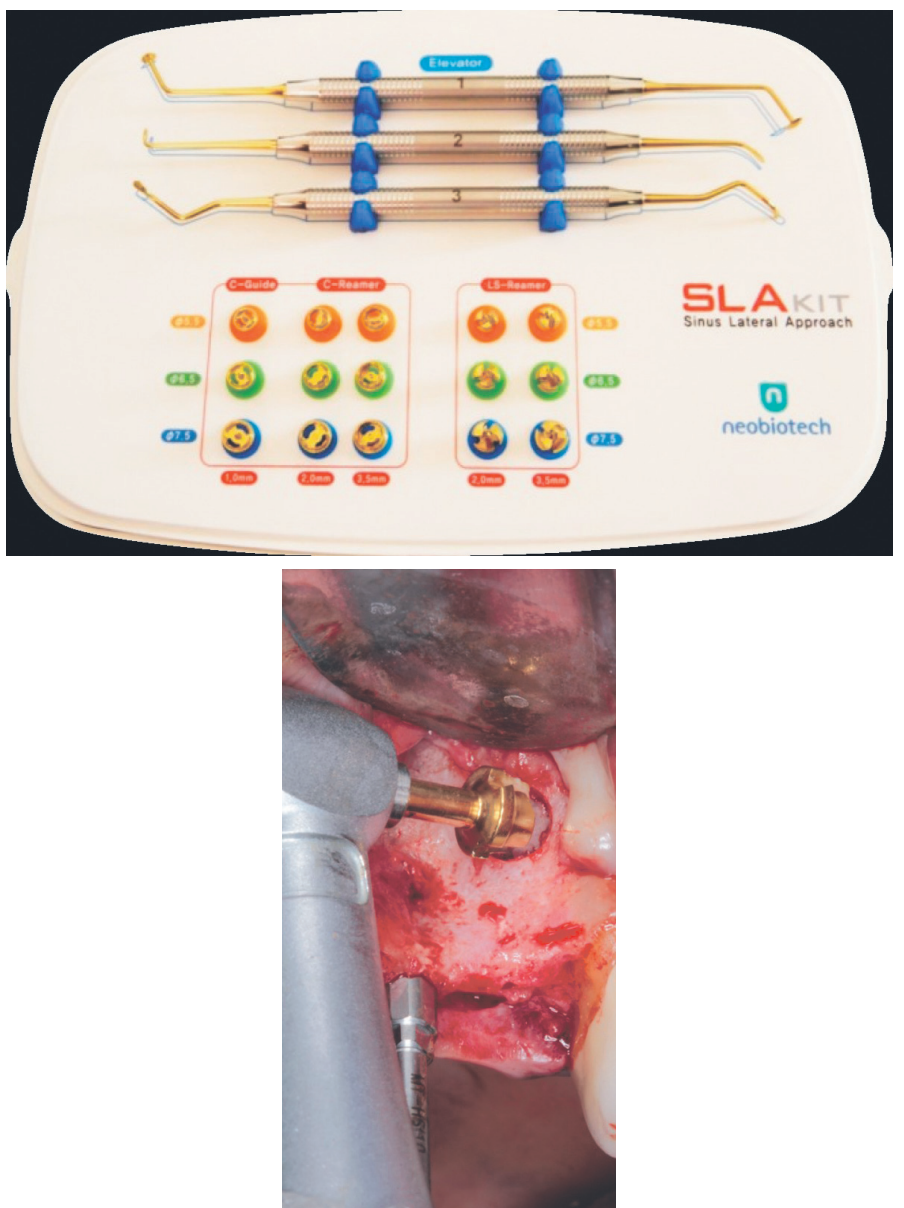

Figura 2. Fig 2a. Se muestra el SLA kit de NeoBiotech. Fig 2b:Fotografía del momento de fresado. Se puede observar la posición del pin de paralelismo del diente 24 y la formación de la ventana lateral de $5.5 \mathrm{~mm}$ de diámetro.

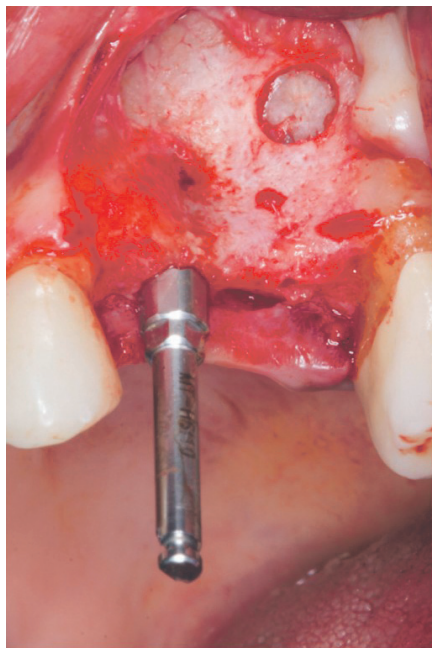

Figura 3. Se muestra la ventana lateral después de la osteotomía dejando expuesta la membrana sinusal.

ventana lateral introduciendo la fresa hasta desgastar la cortical ósea y dejar visible la membrana sinusal. (Figura 3 )

Después de realizar la osteotomía, se continuó con el desprendimiento de la membrana de sinusal con los instrumentos curvos específicos presentes en el kit, colocándolos sobre la parte interna de la cortical vestibular para elevar la membrana sin el riesgo a perforarla. (Figura $4 a$ y fig $4 b)$

Una vez elevada la membrana, se rellenó la cavida ósea con material de injerto. El material de elección en este caso fue $1 \mathrm{gr}$ de xenoinjerto de 

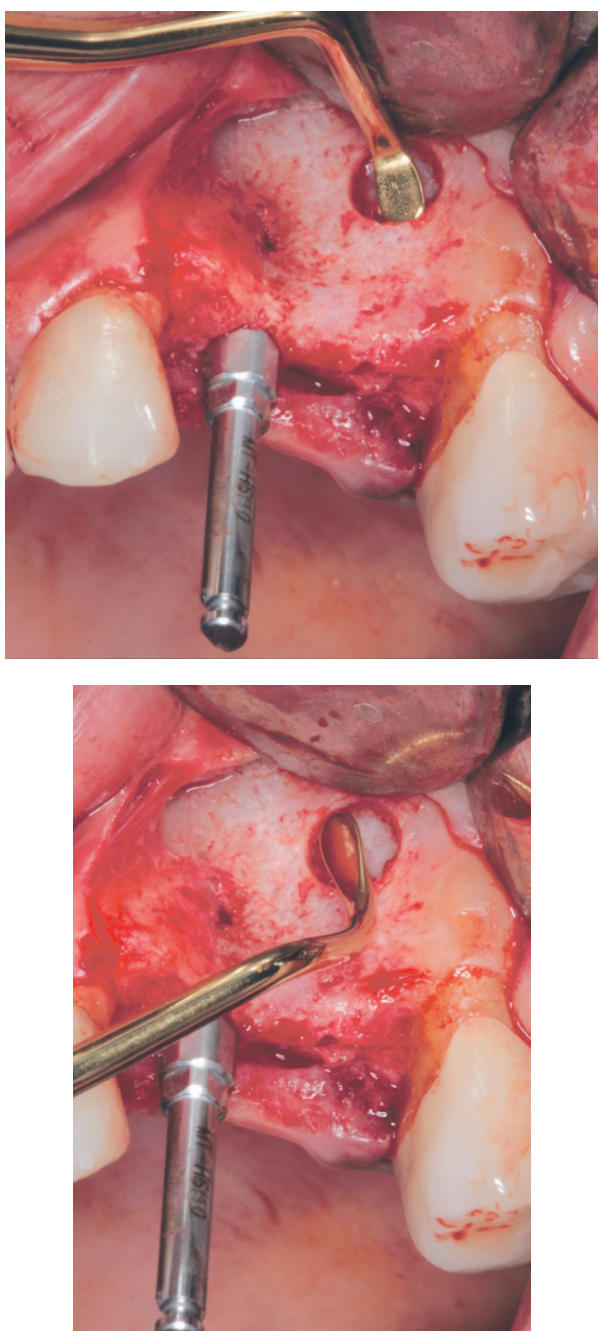

Figura 4a y 4b. Se realiza el desprendimiento de la membrana sinusal.

partícula $0.25 \mathrm{~mm}-1.0 \mathrm{~mm}$ que se colocó hasta rellenar por completo la cavidad ósea. (Figura $5 a$ )

Posteriormente se fresó para la colocación del implante del diente 26. Después del fresado, se continuó con la colocación de implantes de $3.75 \mathrm{~mm} \times 11.5 \mathrm{~mm}$ en la zona del diente 24 y de $4.2 \mathrm{~mm} \times 11.5 \mathrm{~mm}$ en la zona del diente 26 . (Figura $5 b$ )

Al finalizar la colocación de los implantes, se continuó con la liberación del periostio para lograr el cierre primario de la herida y se suturó con ácido poliglicólico 5-0. (Figura 6a) Se tomó una radiografía post operatoria presentando una buena ubicación de los implantes y la correcta elevación de la membrana sinusal. (Figura 6b)

Los resultados obtenidos se valoraron al momento de retirar los puntos de sutura, es decir, a los 14 días, donde se pudo observar una clínica epitelización de la herida. No hubo exposición de los implantes y la paciente reportó pocas molestias postoperatorias

Después de los 6 meses se tomó radiografía de control mostrando la zona del injerto subantral completamente densa y compacta, sin presencia de áreas radiolúcidas o secuestros óseos. La paciente se programó para la rehabilitación protésica. (Figura 7)

\section{DISCUSIÓN}

La elevación de piso de seno maxilar con injerto subantral es un procedimiento quirúrgico que en la actualidad se considera uno de los métodos más comunes y aceptados para aumentar el volumen óseo en la zona del maxilar posterior para la colocación de implantes. Los reportes de la literatura demuestran un alto porcentaje de éxito al usar esta técnica, convirtiéndola en un procedimiento predecible, efectivo y seguro. ${ }^{(4)}$

Misch desarrolla una clasificación basándose en la altura ósea residual subantral para tratar el maxilar posterior edéntulo. Cada una de estas categorías se subdivide en dos en función de la anchura ósea crestal: la división A, que tiene un hueso de $5 \mathrm{~mm}$ de ancho o mayor y
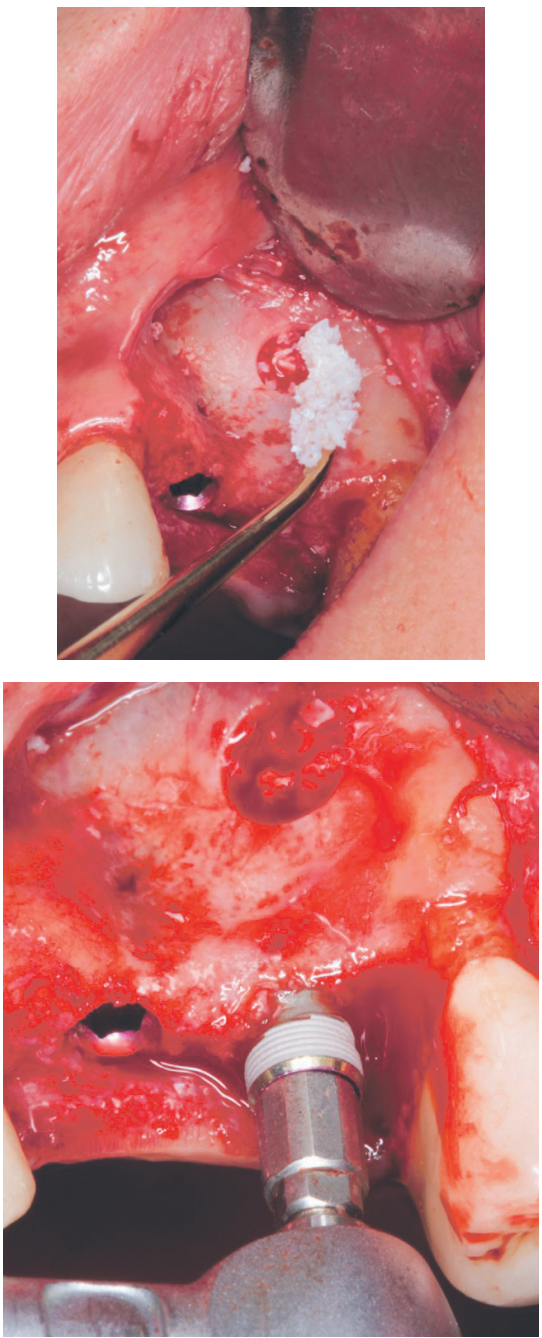

Figura 5. Fig 5a: Muestra la colocación del injerto. Fig 5b:Se observa la colocación de implantes 3.75 x $11.5 \mathrm{~mm}(24)$ y 4.2 x $11.5 \mathrm{~mm}(26)$

la división $B$ que tiene un hueso crestal de entre 2,5 y $5 \mathrm{~mm}$ de ancho. ${ }^{(5)}$ Nuestro paciente al tener un reborde residual de $4.6 \mathrm{~mm}$ se encontraba en la división $B$.

Existen muchos protocolos quirúrgicos y modificaciones para esta técnica. La técnica de la elevación del piso del seno maxilar con osteotomía lateral tiene dos variantes respecto de la colocación de los implantes. La primera es la llamada técnica en dos tiempos quirúrgicos, en la cual en un primer tiempo se realiza la elevación del piso del seno maxilar y luego de un tiempo de espera de 3 y 6 meses. La segunda es llamada técnica en un mismo tiempo quirúrgico, en la cual los implantes son colocados de forma simultánea con la elevación del piso de seno.

Esto fundamentalmente determinado por el espesor del reborde residual. Cuando la altura del piso del seno es inferior a $4 \mathrm{~mm}$ no podemos asegurar una estabilidad primaria por lo tanto se utilizaría la primer variante colocando los implantes de forma diferida. ${ }^{(6)}$

Debido a que la altura del reborde de dicho paciente era superior de $4 \mathrm{~mm}$ optamos por la colocación simultánea de los implantes, lo cual nos da una gran ventaja en cuanto al tiempo total de la restauración.

Existe una amplia variedad de materiales de injerto óseo subantral y hoy en día el estándar de oro sigue siendo el hueso autólogo, ya que no sólo provee células osteoblásticas sino también confiere osteoinducción y osteoconducción ofreciendo matrices orgánicas e inorgánicas y células óseas viables sin riesgo de antigenicidad. Sin embargo, v la cantidad de hueso obtenido intraoralmente es generalmente insuficiente para el aumento de piso de seno maxilar bilateral. ${ }^{(7)}$

Del Fabro et al. realizó un estudio en el cual evaluaron el rango de supervivencia de implantes colocados en senos maxilares que habían sido rellenados con distintos injertos óseos y en donde intentaron estudiar cómo influye el material relleno, la superficie del implante y la técnica quirúrgica sobre el rango de supervivencia de los implantes, demostró que los injertos autógenos combinados tenían una supervivencia del implante del $94,88 \%$ y otros sustitutos óseos, del $95,98 \%$; por lo tanto 

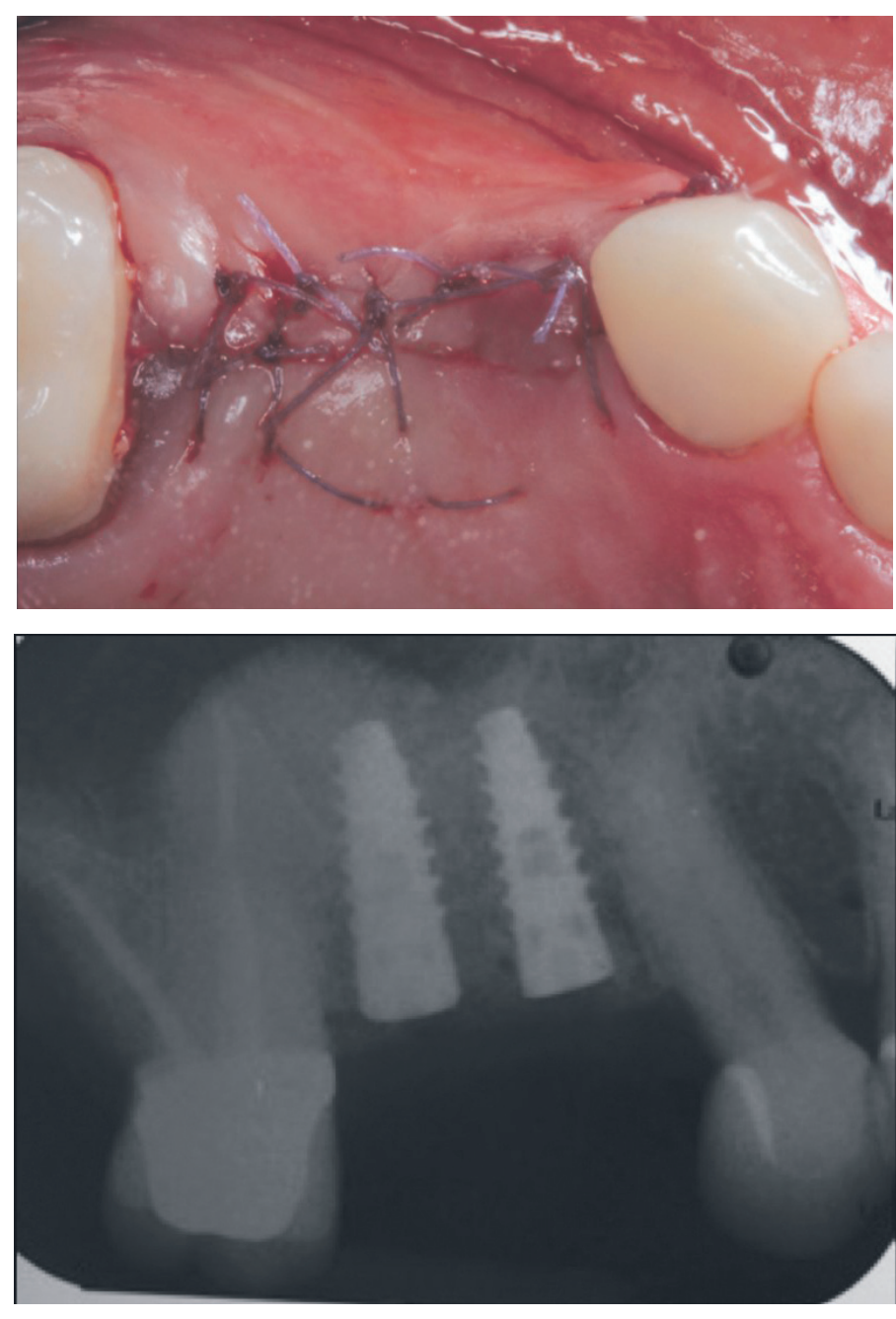

Figura 6. Fig 6a. Se muestra el cierre primario de la herida suturado con ácido ploglicólico 5-0. Fig 6b: Se observa la radiografía post quirúrgica con la elevación del piso de seno y la colocación de los implantes.

se decidió colocar un material de relleno xenoinjerto debido a la facilidad para obtenerlo. ${ }^{(8)}$

Por lo tanto, muchos autores defienden el uso de materiales obtenidos de otros orígenes (aloinjertos, xenoinjertos y aloplásticos) en combinación con el hueso autólogo, argumentando además que existe un tiempo de

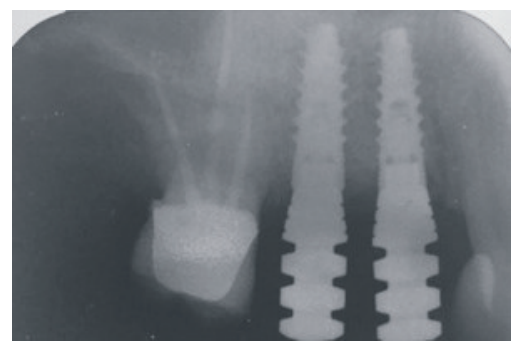

Figura 7. Radiografía de control a los 7 meses.

reabsorción más apropiado. La elección del material o la mezcla de éstos depende muchas veces de la cantidad ósea requerida.

La técnica de ventana lateral está fuertemente relacionada a complicaciones tales como perforación de la membrana y sinusitis. Romanos describe una técnica diferente para la preparación de la ventana lateral. La describe utilizando una fresa de bola con suficiente irrigación de solución salina para realizar la ostetotomía. ${ }^{(9)}$

Sohn et al, realizaron la osteotomía utilizando distintos tipos de láser en donde se encontró una tasa de perforación de $33.3 \%$. Varcellotti demostró que existe una tasa de perforación de $11.1 \%$ utilizando un abordaje ultrasónico. ${ }^{(10,11)}$

EI SLA kit presenta diferencias de una trefina normal. La periferia del área de contacto de la trefina es curva por lo que se mantiene en contacto con la cortical ósea. La técnica descrita en este artículo presenta una serie de ventajas tales como la reducción del tiempo quirúrgico, una pequeña y precisa área de acceso para la elevación de seno y un menor riesgo de perforación de la membrana sinusal. Debido a que el kit requiere la misma pieza de mano que para la colocación de implantes, se elimina el uso de distintas piezas de mano o piezo eléctrico, reduciendo asi el costo quirúrgico.(12)

Es importante considerar que este procedimiento quirúrgico requiere de un minucioso plan de tratamiento y el conocimiento extenso, habilidad y experiencia del cirujano. ${ }^{(13,14)}$

\section{RESPONSABILIDADES ÉTICAS}

Protección de personas y animales. Los autores declaran que para esta investigación no se han realizado experimentos humanos ni en animales.

\section{CONFIDENCIALIDAD DE DATOS.}

Los autores declaran que en este artículo no aparecen datos de pacientes.

\section{DERECHO A LA PRIVACIDAD Y CONSENTIMIENTO INFORMADO.}

Los autores declaran que en este artículo no aparecen datos de pacientes.

\section{Bibliografía}

1. Peleg M, Mazor Z, Garg AK. Augmentation grafting of the maxillary sinus and simultaneous implant placement in patients with 3 to $5 \mathrm{~mm}$ of residual alveolar bone height. Int J Oral Maxillofac Implants. 1999; 14: 549-556

2. Fugazzotto PA, Vlassis J. Long-term success of sinus augmentation using various surgical approaches and grafting materials. Int J Oral Maxillofac Implants. 1998; 13:52-8

3. Jurisic M, Markovic A, Radulovic M, Brkovic BM, Sdndor GK. Maxillary sinus floo augmentation: comparing osteotome with lateral window immediate and delayed implant placements. An interim report. Oral Surg Oral Med, Oral Pathol, Oral Radio Endod. 2008; 106:820-7.

4. Wallace S, Froum S. Effect of maxillary sinus augmentation on the survival of endosseous dental implants. A systematic review. Ann Periodontol. 2003; 8: 328-343 5. Misch C. L' Odontoiatria Implantare Contemporanea. Roma: Antonio Delfino Editores; 2000

6. Kahnberg K, Vannas L. Sinus lift procedure using 2 stage surgical technique I: Clinical and radiographic report up to 5 years. Int J Oral Maxillofac Implants. 2008; 23(5): 876-84

7. Mellonig J. Autogenous and allogenic bone grafts in periodontal therapy. Crit Rev Oral Biol Med. 1992; 3 (4): 333-352
8. Del Fabbro M, Testory T, Francetti L,Weinstein R. Systematic Review of Survival Rates for Implants Placed in the Grafted Maxillary Sinus. Int J Periodontics Restorative Dent. 2004; 24: 565-577

9. Romanos GE. Window preparation for sinus lift procedures: a simplified technique. Implant Dent. 2008;17:377-81

10. Stacchi C, Varcellotti T, Toschetti A, Speroni S, Salgarello S, Di Lenarda R Intraoperative complications during sinus floor elevation using two diferente ultrasonic approaches: a two center, randomized controlled clinical trial. Clin Implant Dent Relat Res. 2015; 17 Suppl 1:e117-25.

11. Sohn DS, Lee JS, An KM, Romanos GE. Erbium, chromium:yttrium-scandiumgallium-garnet laser-assisted sinus graft procedure. Lasers Med Sci. 2009;24:673-7. 12. Farré-Pagés N, Augé-Castro ML, Alaejos-Algarra F, Mareque-Bueno J, FerrésPadró $\mathrm{E}$, Hernández-Alfaro F. A novel trephine design for sinus lift lateral approach Case report. Med Oral Patol Oral Cir Bucal. 2011 Jan 1;16 (1):e79-82

13. Kan $\mathrm{J}$ et al. Factors affecting the survival of implants placed in grafted maxillary sinuses: a clinical report. J Prosthet Dent. 2002; 87: 485-489

14. Pjetursson B, Ching Tan W, Zwahlen M, Lang N. A systematic review of the success of sinus floor elevation and survival of implants inserted in combination with sinus floor elevation. J Clin Periodontol. 2008; 35 (Suppl. 8): 216-240. 